\title{
The utilities of the therapeutic intervention scoring system (TISS-28)
}

\author{
Seetharaman Hariharan, Deryk Chen, Lorna Merritt-Charles, Nahmorah Bobb, Loren DeFreitas, \\ Joann Mohamed André Esdelle-Thomas, Delise Charles, Karen Colley, Elise Renaud
}

Background and Aims: The study evaluates the utility of therapeutic intervention scoring system (TISS28) in quantifying the resource utilization, costs and predicting outcome of critically ill patients. Materials and Methods: TISS-28 was prospectively applied to patients consecutively admitted to the intensive care units (ICU) of three public teaching hospitals and two private hospitals in Trinidad on a daily basis for a period of eight weeks. Demographic data, diagnoses on admission, nurse-patient ratio, ICU length of stay and hospital outcomes were recorded. Simplified acute physiology score (SAPS)-II was applied for all adult patients. Costs were calculated from data collected from the public hospitals in relation to TISS-28 score. Results: TISS-28 scores of five hundred and ninety-five patient-days were analyzed. The median daily TISS28 per patient was 27 [24.5, 30.6 quartiles (IQR)]; the median day- 1 TISS-28 score was 29 (25, 33 IQR) and the median last day TISS-28 score was 25 (21, 30 IQR). The overall average TISS per nurse was 26.2 per day. The mean cost per patient per day was 414 US dollars. The discriminatory function of day- 1 TISS-28 as a prognostic scoring system was less compared to SAPS II as shown by the area under the receiver operating characteristic curve ( 0.65 compared to 0.71 ). Conclusions: TISS-28 is useful for evaluating the resource utilization and costs and may not be useful as a prognostic scoring system

Key words: Intensive care units costs, resource utilization, therapeutic intervention scores

\section{Introduction}

Critical care has evolved as a specialty of its own in both the developed and developing countries consuming a large part of the healthcare budget. ${ }^{[1,2]}$ In recent years there has been an increase in the need for intensive care units (ICU) with more aggressive therapeutic procedures and/or for the increased use of adequate and invasive treatment for advanced diseases ${ }^{[3]}$ Due to the high-

\section{From:}

Anaesthesia and Intensive Care Unit, Faculty of Medical Sciences,

The University of the West Indies, St. Augustine, Eric Williams Medical

Sciences Complex, Trinidad and Tobago, West Indies

\section{Correspondence:}

Dr. Seetharaman Hariharan, The University of the West Indies, Eric Williams Medical Sciences Complex, Mount Hope, Trinidad (West Indies).

E-mail: uwi.hariharan@gmail.com technology care offered in ICUs and the higher cost involved with this, proper evaluation and utilization of the ICU resources should be of very high priority. ${ }^{[4]}$ This is especially true in developing countries such as ours where budgetary allocation has to be prudent to ensure minimal wastage of resources.

The therapeutic intervention scoring system (TISS) is one of the widely applied scoring systems which mostly rely on therapeutic, diagnostic and nursing activities. ${ }^{[5]}$ Besides evaluation of the therapeutic interventions, TISS also assists in quantification of nursing workload, ICU cost evaluation and has been proposed as a valuable tool for analyzing the utilization of ICU resources. ${ }^{[6]}$ Originally 
introduced in 1970, this scoring system comprised of 76 interventions, later updated during 1983. ${ }^{[7,8]}$ Although initially designed to be a prognostic scoring system, the system has been predominantly applied for evaluation of cost and nursing workload. TISS-28, a simplified version of TISS was published in 1996 and is being used as a tool for assessing resource utilization and prognosis after discharge from ICU. ${ }^{[9]}$

With this background the present study aimed at applying this scoring system to evaluate its utility in quantifying the resource utilization, costs and prognosis of the patients in five ICUs in Trinidad and Tobago.

\section{Materials and Methods}

Approval of the Ethics Committee of the University of the West Indies was obtained prior to the study. Approvals were also obtained from the hospital authorities for collection of data. All patients admitted consecutively to the ICUs of three teaching hospitals and two private institutions in Trinidad over a period of eight weeks from July 2005 through August 2005 were included for prospective collection of data.

\section{Hospitals and ICU setting}

Trinidad and Tobago is a twin-island nation of the English-speaking Caribbean, with a population of 1.3 million. The Gross Domestic Product (GDP) (by purchasing power parity) is 18 billion US dollars, with a per capita income of 16,700 US\$. The total annual budget of Trinidad and Tobago is about 6 billion US dollars, of which approximately $6 \%$ is spent on the healthcare sector and the per capita expenditure on health is about 279 US\$ (Pan American Health Organization, 2005). There are three major public hospitals in Trinidad namely the Port-of-Spain General Hospital, Eric Williams Medical Sciences Complex and San Fernando General Hospital, all being teaching hospitals and affiliated to the University of the West Indies. All the ICUs are multidisciplinary units admitting adult medical and surgical patients. The ICU at Eric Williams Medical Sciences Complex is the only unit, which admits pediatric patients. The total number of ICU beds in all the three hospitals is 17 . The private institutions have four-bed and six-bed ICUs respectively, admitting adult patients.

The Radiology Department and the Pathology, Microbiology laboratories of all the hospitals have state-of-the-art equipment facilitating a wide range of investigations. The ICUs in all the hospitals also have facilities for blood gas analyses, portable radiograph and ultrasound.

\section{Data collection}

Demographic data such as the age and gender of the patients, the diagnoses, hospital outcomes and the ICU length of stay were recorded prospectively for all patients. The worst physiological simplified acute physiology score (SAPS) II was recorded on admission to the ICU. ${ }^{[10]}$

TISS-28 was scored on a daily basis from the day of admission to the day of discharge or death. Data were collected from ICU patient charts and files. The patients were followed up throughout their stay in the ICU and the hospital after discharge from the ICU. The nursing staffing pattern for each ICU was evaluated by recording the availability of number of nurses per shift per patient. The TISS-28 per nurse was calculated by dividing the product of the mean daily TISS-28 and the number of patients by the number of nurses in a given ICU.

Data were collected from the Departments of Human Resources, Biomedical Engineering, Accounts and Finance and Pharmacy to calculate the costs for each therapeutic intervention in all the public hospitals. The private hospitals declined to provide information regarding the cost of each therapeutic intervention.

\section{Cost evaluation}

The acquisition costs of drugs and consumables required for each intervention were recorded. This formed the major proportion of the cost for an intervention. The salaries of physicians and nurses were obtained and scaled down to per hour basis and divided among the total number of patients in the ICU. This amount was added to the acquisition costs. Similarly the costs of fixed assets were obtained from the Biomedical Engineering and Finance Departments and appropriate depreciation was calculated. The charges of utilizing the equipment for each intervention were then calculated and added to the total cost for each intervention. The overall cost for each intervention was thus derived. Costs were then assigned to all patients on a daily basis in accordance with the interventions and the TISS-28 score they received each day.

Variables such as predicted mortality, prognostic 
scores, observed mortality rates, therapeutic intervention scores, length of stay, diagnostic categories as well as standard demographics of age and gender were analyzed descriptively to determine their distribution. Predicted mortality for adult patients was calculated by the logistic regression equation published with the SAPS II system.

Wilcoxon ranked sum test was used to compare the age, SAPS II, day-1 TISS-28, last day TISS-28, average daily TISS-28 and length of stay between survivors and nonsurvivors. A two-tailed Pearson's correlation test was used to compare SAPS II score with day-1 TISS-28 and last day TISS-28.

Receiver operating characteristic (ROC) curves were constructed and the area under the curve (AUC) and the 95\% confidence intervals were obtained to measure the ability of the TISS-28 and the SAPS II scoring systems to discriminate between survivors and nonsurvivors.

All data were analyzed using the Statistical Package for Social Sciences, Version 12 (SPSS ${ }^{\circledR}-12.0$ for Windows $^{\circledR}$ ) (Chicago, IL, USA) software. The statistical significance was fixed at the level of $P<0.05$.

\section{Results}

During the two-month period of study, 82 patients were enrolled into the study. 28 patients died during the study period (overall mortality rate $34.1 \%$ ).

Table 1 shows the comparison of the age, ICU length of stay, Day-1 TISS, last day TISS and average daily TISS, between survivors and non-survivors by Mann Whitney- $U$ test. The SAPS-II scores and the predicted mortality were compared. Day-1 TISS-28, last day TISS-28 and average daily TISS-28 were significantly higher in nonsurvivors. However the age and the ICU length of stay did not show any statistically significant difference between survivors and nonsurvivors.

Table 2 shows the distribution of all the patients according to their systemic illnesses and/or etiology during the study period. Majority of patients belonged to the category of respiratory illnesses followed by neurological illnesses and trauma. Overall, 80 (97.5\%) patients received mechanical ventilation, 26 patients received inotropic support, two patients required hemodialysis in the ICU. 62 patients had invasive lines in the form of intra-arterial catheter and central venous line. A total of 595, TISS-scored patient-days were analyzed. The mean TISS-28 score in private hospitals were lower than that of the public hospitals. No patient had pulmonary artery catheter.

A total of 595 TISS-scored patient-days were analyzed. The minimum TISS-28 was 12 and the maximum was 43. The overall average TISS-28 per nurse was 26.2 per day. The mean TISS-28 score in private hospitals were low than that of the public hospitals. Table 3 shows the TISS data in the five ICUs studied. A Tukey test analysis showed that there were statistically significant differences between one of the private institutions and public institutions with respect to day-1 TISS-28, last day TISS-28 and average daily TISS-28 ( $P$ values 0.02 0.01 and 0.02 ).

The minimum cost per patient per day in the public hospitals was 1168.80 Trinidad and Tobago dollars

\section{Table 2: Diagnostic categories of patients}

Diagnostic category by systems

Number (\%)

Respiratory system

Central nervous system

$18(22)$

Multiple trauma

Cardiovascular system

Gastrointestinal system

Poisoning

Renal failure

Others
$16(19.5)$

$15(18.3)$

$10(12.2)$

$5(6.1)$

$4(4.9)$

$2(2.4)$

$12(14.6)$

Table 1: Comparison between survivors and nonsurvivors

Variable
Age
Intensive care unit length of stay (days)
Day-1 TISS
Last day TISS
Average TISS
SAPS II ( $\mathrm{n}=75$ )
Predicted death rate by SAPS II (\%)

Overall
(Median, IQR)
$41.5(22,57.3)$
$5(2,9.8)$
$29(25,33)$
$25(21,30)$
$27.3(24.5,30.6)$
$42(25,56)$

\section{Survivors}

(Median, IQR)

$43(22,57.3)$

$5(2.8,7.3)$

$27(20.7,31.2)$

$21.5(15.8,25)$

$25.3(20.3,27.8)$

$32(24,49)$

$11.7(5.5,42)$

Nonsurvivors
(Median, IQR)
$37.5(22.8,60)$
$6(2,14.8)$
$30(26,34)$
$27(23.3,32.8)$
$30(25.2,32.2)$
$52.5(42,61.8)$
$51.9(28.5,71.4)$

Significance

$P=0.856$ (NS)

$P=0.461$ (NS)

$P=0.025^{*}$

$P<0.001^{\star}$

$P<0.001^{\star}$

$P=0.002^{\star}$

$P<0.001^{\star}$

*Statistically significant by Wilcoxon ranked sum test, TISS: Therapeutic intervention scoring system-28, SAPS II: Simplified acute physiology score -II, IQR: Interquartile ranges, NS: Not significant 


\section{Table 3: TISS-28 data hospital-wise}

\section{INSTITUTION}

Public Hospital I

Public Hospital II

Public Hospital III

Private Hospital I

Private Hospital II

SAPS II score
(Median, IQR)
$53(42,61)$
$35.5(24.7,59.5)$
$43.5(29,56)$
$25(22,49)$
$22(15.5,35)$

SAPS II score
(Median, IQR)

$53(42,61)$

$43.5(29,56)$

$22(15.5,35)$

Day-1 TISS
(Mean \pm SD)

$28.1 \pm 5.9$

$26.4 \pm 10.4$

$30.2+4.9$

$24.9 \pm 5.5$

$20.5 \pm 7.1^{*}$
Last day TISS
(Mean \pm SD)

$25.2 \pm 8.1$

$25.6 \pm 8.2$

$25.8 \pm 5.0$

$21.0 \pm 5.3$

$16.3 \pm 3.8^{*}$
Average TISS per patient

(Mean \pm SD)

$27.1 \pm 5.9$

$24.3 \pm 6.2$

$28.1 \pm 3.7$

$25.6 \pm 1.9$

$18.2 \pm 4.5^{\star}$

*Statistically significant by Tukey test compared to public hospitals, SAPS II: Simplified acute physiological score -II, TISS: Therapeutic intervention scoring system-28, SD: Standard deviation, IQR: Interquartile range

(TT\$) (195 US dollars) and the maximum was 3993.40 TT\$ (665 US\$) and the median costs per patient per day was 2566.50 TT\$ (428 US\$). Because all the public hospitals had uniform salaries and supplies with respect to equipment the cost per TISS point did not vary between the hospitals.

A two-tailed Pearson's correlation test to compare SAPS II with day-1 TISS-28 and last day TISS-28 were

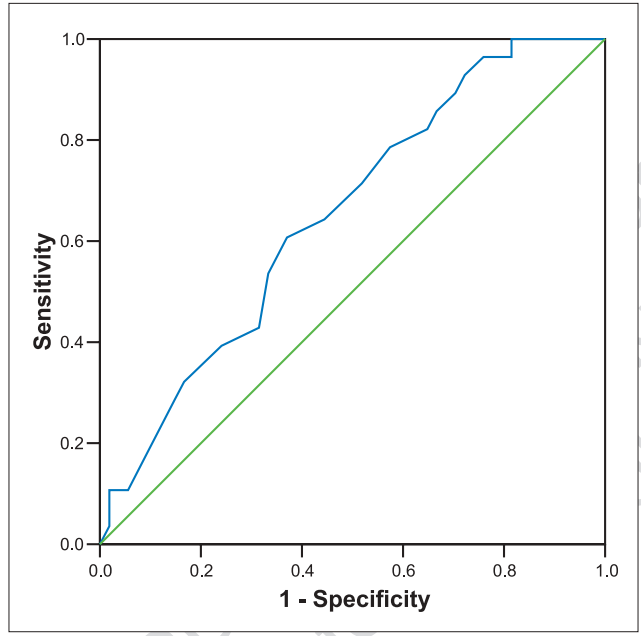

Figure 1: Receiver operating curve for day-1 TISS-28 as an outcome predictor

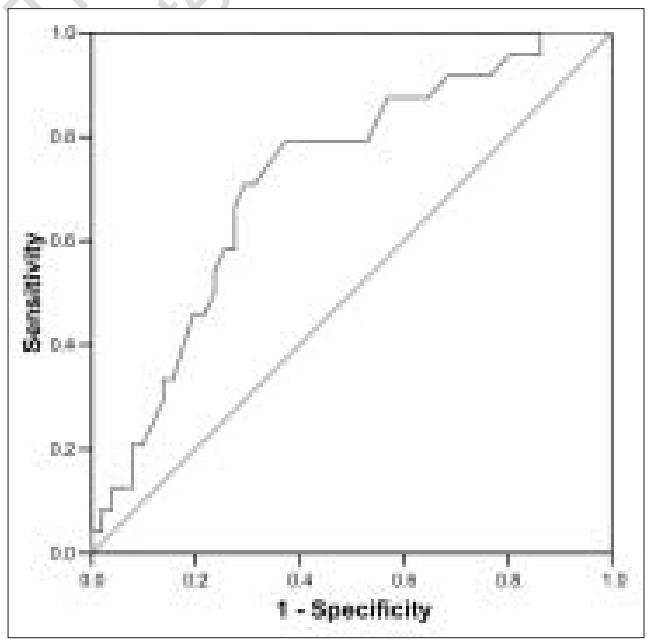

Figure 2: Receiver operating curve for SAPS-II significant at $P<0.01$, although the correlation coefficients $\left(r^{2}\right)$ were low (0.34).

Figure 1 shows the ROC curve to assess the discriminating ability of TISS-28. The area under the curve was 0.65 (95\% confidence intervals $0.53,0.77)$. Figure 2 shows the ROC curve for SAPS II. The area under the curve was 0.71 (95\% confidence intervals $0.59,0.84)$.

Figure 3 shows the trend of the therapeutic intensity of survivors and non-survivors during the last week of the patients' stay in the ICU. The therapeutic intensity did not have a statistically significant decrease in the survivors before discharge.

\section{Discussion}

The present study attempted to evaluate the utility of the TISS-28 in five ICUs in Trinidad and Tobago. The findings of the study suggests that TISS - 28 could be applied to evaluate the resource utilization, process of care and cost evaluation, although it did not perform well as a prognostic model when compared to a physiological scoring system for severity of illness.

Many aspects of the ICU such as the nursing activity

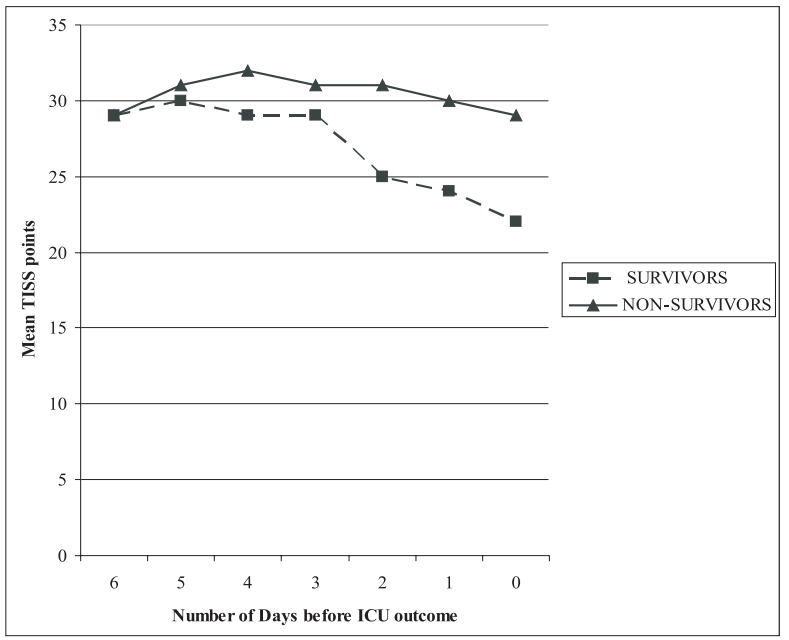

Figure 3: Trends of TISS-28 points during the last week of ICU stay 
and intensity of the therapeutic interventions which are the indirect measures of the process of an ICU may not be able to be assessed with the help of physiological scoring systems. ${ }^{[11]}$ Hence we applied TISS-28 in interdisciplinary ICUs, because it does not depend on the physiological parameters of the patient. The authors who devised the TISS-28 have recommended that the scoring system needs to be validated in multinational ICUs since it was originally validated in Dutch ICUs. ${ }^{[9]}$

TISS-28 has been proposed to assess the nursing activity of a unit indirectly, although this system was further refined to form a Nursing Activity Score (NAS) which claims to perform better than TISS-28 per se to quantify nursing workload. ${ }^{[12]}$ The number of TISS points per nurse is an indirect way of assessing the nursing activity. According to the recommended standards, a nurse should be capable of managing 46 TISS points per shift. ${ }^{[9]}$ In Trinidad, the average TISS points is well within this limit and is comparable to that managed by nurses in a Barbados ICU. ${ }^{[13]}$

Ideally in an ICU, the nurse to patient ratio should be 1:1. However, if the nursing workload is high, some ICUs may have more than one nurse per patient. ${ }^{[14]}$ Although most ICUs in Trinidad have a 1: 1 nursing pattern, at times this decreased. The Task Force of the European Society of Intensive Care Medicine has described three levels of care in the ICU, which enables hospitals to determine the appropriate nurse to patient ratio. ${ }^{115}$ This guideline requires triage of the ICU patients and may create a more flexible staffing pattern and may lead to more efficient utilization of resources, especially in our situation.

The mean day-1 TISS-28 points reported from North America and Europe range from 25 to 30 points. ${ }^{[16,17]}$ The mean day-1 TISS-28 in the present study was 27.9 points, which may imply that the therapeutic interventions in the ICUs of Trinidad may be similar to those of the developed countries. Also, when compared to an ICU in Barbados (17.9), the mean first-day TISS in Trinidad was much higher. ${ }^{[13]}$ The average daily TISS-28 score (26.8) was comparable to a report from Hong Kong (28.4). ${ }^{[18]}$

ICU resource utilization has been one of the most important aspects studied by the application of TISS. ${ }^{[16,17,19,20]}$ Data from the present study helped us to quantify the processes of care such as mechanical ventilation, renal support etc. in each ICU.

TISS-28 has also been previously applied to quantify the costs of ICU. ${ }^{[21-24]}$ The mean cost per patient per day in the present study is approximately 414 US $\$$ which is less than the mean expenditure in another Caribbean country Barbados (743 US\$). ${ }^{[13]}$ The costs of Trinidad ICU are less than that of the developed countries such as the United States and United Kingdom, but higher than other developing nations such as India. ${ }^{[25]}$

The mean day- 1 TISS-28 between survivors and nonsurvivors showed no statistical difference. The TISS-28 scores have been low in private hospitals when compared to public hospitals. There may be many underlying reasons for this finding. Firstly, patients admitted to private ICUs had relatively lesser severity of illness as evidenced by the low SAPS-II scores [Table 3]. Many of these patients belonged to the diagnostic categories such as postoperative admission following elective surgery. Secondly, in public institutions, the care is offered free-ofcost for the citizens of Trinidad and Tobago. Additionally, because all the three public institutions are affiliated to the University of the West Indies, in an academic environment, the therapeutic interventions would have been probably much more aggressive.

Previous studies have examined the ability of TISS-28 to predict prognosis ICU patients and after their discharge from ICU. ${ }^{[26,27]}$ A previous report has also correlated TISS28 scores to SAPS II scores on admission. ${ }^{[18]}$ The present study analyzed if the day-1 TISS-28 score may be used as a prognostic scoring system. The discriminating ability of TISS-28 has been lesser than that of SAPS-II as shown by the area under the ROC curve. The reasons for the poor performance of TISS-28 as a prognostic model may be due to the fact that this score highly relies upon the individual physician choices of therapeutic interventions such as pulmonary artery catheterization and parenteral nutrition. Although a high TISS-28 score on admission may indirectly imply that the patient might have been severely ill, this may not be always necessarily true.

There are many limitations to the present study. Although we had enough TISS-28 scores and patient-days for analysis, the number of patients included in the study was low. This may be a limiting factor for the analysis of the prognosticating ability of the TISS-28. We could 
not get data from private hospitals to itemize the cost for each intervention in that setting. In public hospitals, cost evaluation was done for each therapeutic intervention by deriving the costs with respect to consumables, staffcosts and fixed assets. Despite the fact that this was done in consultation with the Departments concerned, there could have been some errors by this method. But every cost-evaluation methodology in ICUs has its own inherent flaws. ${ }^{[28]}$

\section{Conclusion}

TISS-28 may be useful to evaluate the resource utilization, process of care and costs of an ICU, although it's prognosticating ability is limited.

\section{References}

1. Fisher MM. Critical care: A specialty without frontiers. Crit Care Clin 1997;13:235-43.

2. Bloomfield EL. The impact of economics on changing medical technology with reference to critical care medicine in the United States. Anesth Analg 2003;96:418-25.

3. Barbieri S, Feltracco P, Michieletto E, Basso I, Spagna A, Giron G. Demand and availability of Intensive Care beds. A study based on the data collected at the SUEM 118 Central of Padua from October 1996 to December 2001. Minerva Anestesiol 2003;69:625-39.

4. Miller DH. The rationing of intensive care. Crit Care Clin 1994;10:135-43.

5. Dawson JA. Admission, discharge and triage in critical care- principles in practice. Crit Care Clin 1993;9:555-74.

6. Apostolopoulou E, Nikoloudi P, Georgoudi E, Veldekis D. Utilization of intensive care unit: A multicentre study in Greece. ICU Nurs Web J 2005;23:1-6.

7. Cullen DJ, Civetta JM, Briggs BA, Ferrara LC. Therapeutic intervention scoring system: A method for quantitative comparison of patient care. Crit Care Med 1974;2:57-60.

8. Keene AR, Cullen DJ. Therapeutic intervention scoring system: Update 1983. Crit Care Med 1983;11:1-3.

9. Miranda DR, de Rijk A, Shaufeli W. Simplified therapeutic intervention scoring system: The TISS-28 items. Crit Care Med 1996;24:64-73.

10. LeGall JR, Lemeshow S, Saulnier F. A new Simplified Acute Physiology Score (SAPS II) based on a European/North American multicenter study. JAMA 1993;270:2957-63.

11. Hariharan S, Dey PK, Chen DR, Moseley HS, Kumar AY. Application of Analytic Hierarchy Process for measuring and comparing the global performance of intensive care units. J Crit Care 2005;20:117-25.

12. Guccione A, Morena A, Pezzi A, lapichino G. The assessment of nursing workload. Minerva Anestesiol 2004;70:411-6.
13. Hariharan S, Moseley HS, Kumar AY. Outcome evaluation in a surgical intensive care unit in Barbados. Anaesthesia 2002;57:434-41.

14. Edbrooke D, Hibbert C, Corcoran M. Review for the NHS executive of adult critical care: An international perspective. [Last accessed on 2005 Jul]. Available from: http//:www.dh.gov.uk/assetRoot/ 04/10/84/48/04108448.pdf.

15. Ferdinande P. Recommendations on the minimal requirements for the Intensive Care Departments. Members of the Task Force of the European Society on Intensive Care Medicine. Intensive Care Med 1997;23:226-32.

16. Byrick R, Caskennette GM. Audit of critical care: Aims, use, cost and limitations of a Canadian system. Can J Anaesth 1992;39:260-9.

17. Dragsted L, Qvist J. Outcome from intensive care. I. A 5-year study of 1308 patients: methodology and patient population. Eur $\mathrm{J}$ Anesthesiol 1989;6:23-37.

18. Yee Kwok WW, Chun Chau JP, Pau Le Low L, Thompson DR. The reliability and validity of the therapeutic activity index. J Crit Care 2005;20:257-63.

19. Stricker K, Rothen HU, Takala J. Resource use in the ICU: Short- vs. long-term patients. Acta Anaesthesiol Scand 2003;47:508-15.

20. Graf J, Graf C, Janssens U. Analysis of resource use and cost-generating factors in a German medical intensive care unit employing the Therapeutic Intervention Scoring System (TISS-28). Intensive Care Med 2002;28:324-31.

21. Dickie H, Vedio A, Dundas R, Treacher DF, Leach RM. Relationship between TISS and ICU cost. Intensive Care Med 1998;24:1009-17.

22. de Keizer NF, Bonsel GJ, Al MJ, Gemke RJ. The relation between TISS and real paediatric ICU costs: A case study with generalisable methodology. Intensive Care Med 1998;24:1062-9.

23. Moran JL, Peisach AR, Solomon PJ, Martin J. Cost calculation and prediction in adult intensive care: A ground-up utilization study. Anaesth Intensive Care 2004;32:787-97.

24. Parviainen I, Herranen A, Holm A, Uusaro A, Ruokonen E. Results and costs of intensive care in a tertiary university hospital from 1996-2000. Acta Anaesthesiol Scand 2004;48:55-60.

25. Parikh CR, Karnad DR. Quality, cost and outcome of intensive care in a public hospital in Bombay, India. Crit Care Med 1999;27:1754-9.

26. Beck DH, McQuillan P, Smith GB. Waiting for the break of dawn? The effects of discharge time, discharge TISS scores and discharge facility on hospital mortality after intensive care. Intensive Care Med 2002;28:1287-93.

27. Fortis A, Mathas C, Laskou M, Kolias S, Maguina N. Therapeutic Intervention Scoring System-28 as a tool of post ICU outcome prognosis and prevention. Minerva Anestesiol 2004;70:71-81.

28. Gyldmark M. A review of cost studies of intensive care units: Problems with the cost concept. Crit Care Med 1995;23:964-72.

Source of Support: Nil, Conflict of Interest: None declared 